\title{
A lack of interference effects in recognition memory
}

\author{
GORDON B. WILLIS and BENTON J. UNDERWOOD \\ Northwestern University, Evanston, Illinois
}

\begin{abstract}
In two recognition experiments, subjects' study of a list of 50 words was followed by interpolated learning in which (1) the length of time required to present a given number of interpolated words varied and (2) the number of interpolated words varied but the time was held constant. Forced-choice recognition of the original 50 study words occurred immediately after interpolated learning. In the first experiment, there was some evidence that forgetting (between 3 and $12 \mathrm{~min}$ ) was due to the number of interpolated items (interference). The second experiment dealt with retention interval between 0 and $3 \mathrm{~min}$. Forgetting was observed across the $3 \mathrm{~min}$, but there was no evidence that the number of interpolated items influenced this forgetting.
\end{abstract}

The present research was prompted by the previous work of others who have found a lag effect in singleitem recognition-learning studies. This effect surfaces in studies that have used the continuous recognition procedure, in which a subject is presented a long study list and decides at each appearance of an item whether that item has been presented previously in the list. Hockley (1982), Martin and Melton (1970), Shepard and Chang (1963), and Shepard and Teghtsoonian (1961) have used this technique to show that as the lag, or the number of items in the list between separate occurrences of the same item, is increased, recognition performance for the second occurrence decreases.

One is able to interpret studies that use continuous recognition procedures in a way that favors either of two possible explanations. Because the experimenter uses a constant rate of stimulus presentation, as lag is increased, the time between occurrences of an item as well as the number of items intervening are concurrently increased. Forgetting thus cannot be attributed independently to either the increased retention interval for the critical item or to the increased number of additional items between same-item occurrences.

The above ambiguity led us to study recognition memory in a way that avoided the confounding of the two variables. This was done by independently varying each. A retroactive-inhibition paradigm, in which a constant block of critical stimuli was followed by a variable second set, was employed. Within this second set, the number of items and the rate of item presentation were varied in order to achieve control of the total block duration and of the number of items appearing within that duration. A subsequent series of forced-choice tests between the critical stimuli and new items provided

Requests for reprints should be sent to Gordon B. Willis, Department of Psychology, Northwestern University, Evanston, Illinois 60201. measures of recognition performance under the varied conditions. The number of intervening presentations could in this way be varied within a fixed time period; thus, lag in this experiment was truly defined in terms of the number of interpolated stimuli, rather than as a confound of item and time variables. Performance decrement due to introduced manipulations was then attributable to the number of items, the length of retention interval, or both.

\section{EXPERIMENT 1}

\section{Method}

Lists. Initially, a group of 280 items was selected. All were four-letter words of between 1 and 50 per million frequency (Thorndike \& Lorge, 1944), chosen at random, but subject to the restrictions that all began with consonants and that words that began with each consonant were represented in the same proportion with which they occurred within the population of English four-letter words. These words were distributed among a study list and a test list in the following manner. The study list comprised two sections. The initial section consisted of 50 words serving as the critical, or tested, stimuli, and the second section, labeled the intervening (IV) block, contained 180 items. The test list contained 50 pairs of words. Each test-list word pair included an item from the critical list, placed adjacent to a distractor word not presented previously. These pairs were arranged vertically so that the set of 50 repeated stimuli retained its serial ordering relative to study-list presentation, except that the order of the first 10 was reversed. Horizontally, pairs were ordered randomly with respect to whether the repeated word maintained left- or right-hand position. In sum, study and test lists were ordered so that 50 critical plus 180 additional items constituted the study list and 50 pairs consisting of 1 critical and 1 new item appeared in the test list.

Procedure and Subjects. Four conditions in a $2 \times 2$ design were used in order to vary the retention interval and the number of items intervening between study and test presentations. These were produced through a factorial combination of either 3 or $12 \mathrm{~min}$ of IV-block duration, with 30 or 180 item presentations selected to appear within that block. A 3-min presentation was used in Condition 3-30, in which 30 stimuli were presented for $6 \mathrm{sec}$ each, and in Condition 3-180, in which 180 
stimuli were presented for $1 \mathrm{sec}$ each. A 12 -min presentation was used in the remaining two conditions: $12-30$, in which 30 items were presented for $24 \mathrm{sec}$ each, and 12-180, in which 180 items were presented for $4 \mathrm{sec}$ each. Zero interstimulus interval was approximated by use of microcomputer presentation, enabling true exposure durations of the lengths specified above. Time variation of the IV block was achieved by altering the rate of item presentation. If 180 items were to be displayed, the computer presented the entire IV list of words in random order. If 30 words were to be displayed, the program randomly selected these 30 from the entire group of 180 items.

The subjects were 120 Northwestern University undergraduates from an introductory psychology class. Thirty subjects were assigned to each of the four conditions by a block-randomized schedule. Each subject was run individually. The subject was seated at a desk supporting the computer and instructed that he or she would be presented a list of words and then be given a recognition test of those words. The study list was then presented. The critical set of 50 was presented at $3 \mathrm{sec}$ per stimulus, filling a 2.5 -min time block. At the termination of this block, and without warning to the subject, the computer began presentation of the IV block of stimuli. When the appropriate number of these stimuli had been presented, a pause of approximately $20 \mathrm{sec}$ was used in order to explain the nature of the recognition test. The test list of pairs was then presented at a 3 -sec rate in order to equate retention interval for each repeated stimulus. The subjects were to make a choice within this $3 \mathrm{sec}$. This was done by reading aloud the pair item that had been seen previously. If a subject failed to respond to any number of the 50 pairs, he or she was retested on those pairs immediately upon termination of the test block.

\section{Results}

Table 1 shows the mean percentages of correct forcedchoice decisions for the four conditions. Perhaps the most striking fact to be inferred from these data is the lack of substantial differences in performance, the means varying from $78.2 \%$ to $83.9 \%$. Nevertheless, an analysis of variance of the four conditions showed that the effect of the number of intervening items was a reliable factor $[F(1,116)=5.76, p<.02]$. The length of the retention interval was not a significant variable $(F=1.78)$, and the interaction of the two variables produced $\mathrm{F}<1$.

\section{Discussion}

In the above experiment, we held the length of the retention interval constant while varying the number of interpolated items. These manipulations are not without problems. The fewer the items presented, the greater is the duration of exposure of each item and thus, perhaps, the higher the level of learning. It could be supposed further that the higher the level of learning of an item, the greater the interference it would produce. It seemed necessary, therefore, to determine the influence of the rate of presentation (duration of exposure) on recognition performance. This determination is simply made from the present data. If the duration of exposure were a critical variable, recognition

Table 1

Percent Correct Recognition Performance and Standard Deviations in Experiment 1

\begin{tabular}{ccc}
\hline Condition & Mean & SD \\
\hline $3-30$ & 83.9 & 7.0 \\
$3-180$ & 81.0 & 8.5 \\
$12-30$ & 82.6 & 8.5 \\
$12-180$ & 78.2 & 9.3 \\
\hline
\end{tabular}

scores should have been poorer with the 12-min retention interval than with the 3-min interval. The data of Table 1 show that this was not the case. Neither the length of the retention interval per se nor the duration of presentation of the interpolated items (which increased as the retention interval increased) influenced recognition.

One possible explanation for the absence of strong effects in Experiment 1 is that interpolation of varying numbers of items, or variation of the length of the retention interval, produced no forgetting. That is, the $80 \%$ level of responding observed could be said to represent the degree of learning of the critical block of items. This implies that if a test were given immediately following presentation of the critical block of words, recognition scores would be much the same as those given in Table 1 . We conducted a subsidiary experiment to evaluate this possibility. Items selected from those employed in Experiment 1 were presented to 12 subjects for study, and the recognition test was given immediately after the last study word had been shown. For reasons not germane to the present discussion, 48 (instead of 50) words were used. The 12 subjects produced a mean correct score of $93.0 \%$, a value about $11 \%$ higher than the general performance levels revealed in Experiment 1. This indicates that some forgetting did occur in Experiment 1. Experiment 2 represented a further attempt to try to understand the cause of the forgetting.

\section{EXPERIMENT 2}

In Experiment 1, it was found that the addition of $3 \mathrm{~min}$ of rest, of 30 additional items, or possibly of both resulted in a performance level that did not fall appreciably even when the interval was increased to $12 \mathrm{~min}$ or to 180 additional items. The influence of the areas of between 0 and $3 \mathrm{~min}$ of lag time and 0 and 30 additional items had as yet been unexplored. Experiment 2 was designed to fill this gap and used a technique similar to that of Experiment 1, but with different parameters.

\section{Method}

Lists. The stimuli were identical to those used in Experiment 1, and a list similar in structure to that of Experiment 1 was assembled. The list contained 50 initial items, followed by 30 that served as potential interfering items and, finally, by 50 pairs consisting of the initial 50 stimuli along with new words. Initial study and test sections from the Experiment 1 list were retained without change, and the IV block of this new list contained 30 items randomly selected from the IV block of the first list.

Procedure and Subjects. The basic procedure of Experiment 1 was followed, except for changes in the number of conditions and in the values of time and item number used. The conditions employed were as follows. Either 0,15 , or 30 items were presented in the IV block. This block was of either 0-, 1.5-, or 3-min duration. A factorial combination of these variables produced seven unique conditions. Three of these presented 0 items, for time intervals of either $0,1.5$, or $3 \mathrm{~min}$. Four additional conditions were formed by the combination of either 15 or 30 items presented in a block of 1.5 or $3 \mathrm{~min}$. Thus, using the same designation as in Experiment 1, the conditions were $0-0,1.5-0,3-0,1.5-15,1.5-30,3-15$, and 3-30.

The first 50 items were presented at a 3-sec rate, as in Experiment 1 , and the intervening stimuli were presented at rates that filled the given interval with the appropriate number of items. Conditions 1.5-15 and 1.5-30 therefore presented items at rates of 6 and $3 \mathrm{sec}$, respectively, and Conditions 3-15 and 3-30 presented items at rates of 12 and $6 \mathrm{sec}$, respectively.

IV block presentation was determined as follows. If 0 items were to be presented, the subject carried out simple arithmetic tasks for the block duration, in order to prevent rehearsal of 
study items. If 30 items were given in this interval, the computer presented the entire IV block in random order. If 15 items were presented, these were randomly selected from the 30 items of the IV block. Subsequently, the subject made a choice between critical-stimulus words and new words, as in Experiment 1. Again, a 3-sec forced-choice test was used. Fifteen subjects from an introductory psychology class were assigned to each of the seven conditions.

\section{Results}

Mean correct choices for all conditions are plotted in Figure 1. Conditions presenting 15 and 30 items are combined in Figure 1. Correct responding can be seen to decrease linearly over the $3 \mathrm{~min}$ of interpolated time, for all values of item-presentation number. The addition versus nonaddition of IV items appears to have had little effect, in that performance appears to have been about the same when there were 0 interpolated items and when 15 or 30 words were added.

Because of the incomplete factorial nature of the design, statistical analysis was performed through the method of orthogonal contrasts. The effect due to the use of an interpolated retention interval prior to testing is represented by the contrast of the mean of Condition 0-0 with the appropriately weighted sum of the means of the other conditions. This effect was found to be significant $[F(1,98)=4.95, p<.05]$. An effect due solely to retention interval was thus indicated. The effect of varying the length of the retention interval from 1.5 to $3 \mathrm{~min}$ was marginally significant $[F(1,98)=$ $3.41, \mathrm{p}<.07]$. Main effects of item-presentation number were also considered. The difference between performances for conditions of $0 \mathrm{IV}$ items and those of either 15 or 30 items, or the contrast representing the use versus nonuse of IV-block stimuli, was not found to be significant at the .05 level. Finally, the interaction between IV-block duration and use versus nonuse of items within that block also failed to reach significance $(\mathrm{F}<1.0)$.

As in Experiment 1, analysis was done to determine

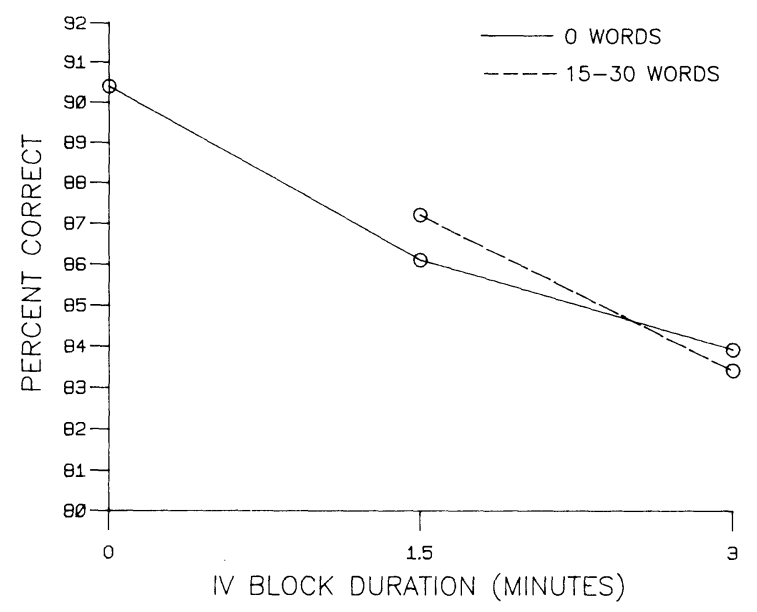

Figure 1. Mean recognition performance in Experiment 2 as a function of IV-block size and duration. the degree of contamination of the results due to the confounding of rate (duration) of presentation of the IV words. Again, the differences over retention interval for those conditions that used IV blocks filled with items are due to both retention interval alone and effects of differential IV-item learning resulting from unequal exposure durations across conditions. Therefore, part of the drop in critical-item performance seen between 1.5 and 3 min may have been due simply to this confound. It may be pointed out, however, that no rate confounding was involved in those conditions presenting zero items within IV blocks, and these produced forgetting with time that is therefore unattributable to rate differences. Thus, this decrease is due to retention interval alone, and any effects due instead to presentation rate in the other conditions would be reflected by an additional drop in slope for those conditions between 1.5 and $3 \mathrm{~min}$. However, little such increased drop is apparent in Figure 1, and no statistical interaction between retention interval and number of items was observed. It therefore seems reasonable to conclude that a factor or factors associated with the retention interval alone produced the forgetting observed in all conditions.

\section{GENERAL DISCUSSION}

Experiment 2 was prompted by the desire to discover whether the addition of items or of time produced the forgetting observed over the first $3 \mathrm{~min}$ of interpolated retention interval. Clearly, time was supported as the critical variable. The effects produced by the number of items were in a direction inconsistent with the expectation of an interference viewpoint, whereas performance decrements over time were clear and statistically significant. One might argue that the arithmetic task produced forgetting in Experiment 2 because it was a source of interference. However, any theory of interference that emphasizes the similarity of the interpolated to the original learning task would have to predict that more forgetting would be found due to the interpolation of words than to the interpolation of an arithmetic task. This was not observed.

Few tests of the mechanisms by which forgetting occurs in recognition have actually been carried out. Evidence is accumulating, however, that interference is not especially important. Several previous studies have failed to discover substantial interference effects in recognition (Bower \& Bostrom, 1968; Underwood \& Brown, 1975). Underwood and Brown, for example, found almost identical forgetting rates for 30 tested pairs over a retention interval of $120 \mathrm{sec}$, independently of the number of preceding lists. Shulman (1974) varied list length from 25 to 100 items and kept the retention interval for tested words constant at about $5 \mathrm{~min}$, and found no evidence for retroactive interference, although some proactive interference was suggested. Recently, Bowles and Glanzer (1983) reported the occurrence of both proactive and retroactive interference in a recognition study. However, as in the present investigation, the magnitudes of the effects were quite small, especially for the retroactive-interference condition.

A simple alternative to interference that may be concluded to have been supported by the results of Experiment 2 is tracedecay theory. However, trace decay has long been regarded as an insufficient explanation of forgetting phenomena. It is clear that decay, as a theoretical construct, is not unequivocably supported by the types of findings uncovered here. Underwood, Zimmerman, and Freund (1971) concluded that a decay conception constitutes little more than reiteration of the data. That item loss 
was found in Experiment 2 to occur over time, independently of item interpolation, seems to indicate that a truly important operative mechanism that varies with time has not been uncovered.

In summary, Experiment 1 revealed some evidence that the number of interpolated words influences recognition, as would be expected from interference theory. However, the effect was small in an absolute sense, and there was no evidence of interference in Experiment 2. The data suggest that the initial loss in recognition memory is not due to the number of interpolated words (Experiment 2), whereas with longer intervals (Experiment 1 ), the number of intervening words may produce a small effect.

\section{REFERENCES}

Bower, G.H., \& Bostrom, A. Absence of within-list PI and RI in short-term recognition memory. Psychonomic Science, 1968, 10, 211-212.

Bowles, N. L., \& Glanzer, M. An analysis of interference in recognition memory. Memory \& Cognition, 1983, 11, 307-315.

HockLEy, W. E. Retrieval processes in continuous recognition. Journal of Experimental Psychology: Learning, Memory, and Cognition, 1982, 8, 497-512.
Martin, E., \& Melton, A. W. Meaningfulness and trigram recognition. Journal of Verbal Learning and Verbal Behavior, 1970, 9, 126-135.

Shepard, R. N., \& Chang, J. J. Stimulus generalization in the learning of classifications. Journal of Experimental Psychology, 1963, 65, 94-102.

Shepard, R. N., \& TEghtsoonian, M. Retention of information under conditions approaching a steady state. Journal of Experimental Psychology, 1961, 62, 302-309.

Shulman, A. I. The declining course of recognition memory. Memory \& Cognition, 1974, 2, 14-18.

Thorndike, E. L., \& LoRGE, I. The teacher's word book of 30,000 words. New York: Teachers College Press, Columbia University Library, 1944.

Underwood, B. J., \& Brown, A. S. Interference in recognition memory: A replication. Bulletin of the Psychonomic Society, 1975, 5, 263-264.

Underwood, B. J., Zimmerman, J., \& Freund, J. S. Retention of frequency information with observations on recognition and recall. Journal of Experimental Psychology, 1971, 87, 149-162.

(Manuscript received for publication August 4, 1983.) 